\title{
Young ASD children in special and mainstream education settings have similar behavioral characteristics
}

Michal Ilan*1,2,3, Gal Meiri*2,3, Liora Manelis²,3, Michal Faroy ${ }^{2,3}$, Analya Michaelovski4, Hagit Flusser ${ }^{4}$, Hagar Binoun-Chaki ${ }^{4}$, Ronit Segev-Cojocaru ${ }^{3}$, Orly Dotan ${ }^{3}$, Hen Schtaerman ${ }^{5}$, Idan Menashe, ${ }^{2,6}$, llan Dinstein ${ }^{1,2,7}$

1. Psychology Department, Ben Gurion University, Beer Sheva, Israel.

2. National Autism Research Center of Israel, Ben Gurion University of the Negev, Be'er Sheva, Israel

3. Pre-School Psychiatry Unit, Soroka University Medical Center, Beer Sheva, Israel.

4. Zusman Child Development Center, Soroka University Medical Center, Beer Sheva, Israel.

5. Child Development Center, Maccabi Health Services, Beer Sheva, Israel

6. Public Health Department, Ben Gurion University, Beer Sheva, Israel.

7. Cognitive and Brain Sciences Department, Ben Gurion University, Beer Sheva, Israel.

* These authors contributed equally to this study 


\section{Abstract}

Large controversy exists regarding the potential benefits and drawbacks of placing young children with ASD in mainstream versus special education settings. Currently, there are no clear clinical recommendations for making such placement decisions and remarkably little information regarding which educational option is better for ASD children with specific abilities and difficulties. Previous studies, mostly focusing on school-age children, have reported that those with poorer cognitive abilities, more severe ASD symptoms, and more challenging behaviors tend to be placed in special education. Here, we utilized the database at the National Autism Research Center of Israel to determine whether behavioral and socio-demographic characteristics influenced the initial placement of 242 young children, immediately after receiving their ASD diagnosis. We performed these comparisons separately for 1-3-year-old children who were placed in daycare centers and 3-5-year-old children who were placed in pre-school kindergartens. Our analyses revealed surprisingly small differences across the two educational settings. The two main factors that differed significantly across groups were cognitive scores and parent education, which were lower in ASD children placed in special education. However, these differences were of moderate effect size and explained a relatively small percentage of the variability in placement choices (up to 15\%). Indeed, we found remarkable overlap in the characteristics of ASD children across educational settings, which suggests that initial placement decisions are mostly performed regardless of the children's behavioral abilities. Large-scale longitudinal studies that compare outcome across educational settings for children with different behavioral characteristics are, therefore, highly warranted. 


\section{Introduction}

The growing rates of ASD around the world require governments to organize educational settings that can serve large numbers of ASD children in an optimal manner. In many western countries, children with ASD are placed in inclusive mainstream education settings (often with a personal assistant) or in exclusive special education settings, where small groups of children receive specialized care from a relatively large staff of special education professionals. Placement decisions have dramatic financial ramifications, because special education settings are considerably more expensive to create and maintain in comparison to mainstream education settings (Chasson et al., 2007). According to the Israeli Ministry of Education, in 2018 approximately one third of ASD children (3-21-years-old) in Israel were placed in mainstream educational settings while two thirds were in autism-only special education settings.

There is ongoing debate regarding the potential benefits and drawbacks of placing children with ASD in mainstream or special education setting (Ravet, 2011). Previous studies have suggested that children with ASD who are integrated into mainstream educational settings are likely to benefit from exposure to the social communication and behaviors of typically developing children. It has been suggested that this exposure enhances language and social abilities, and encourages the development of academic skills (Harrower and Dunlap, 2001). Indeed, children with ASD who are placed in mainstream educational settings exhibit significant improvements over time in social communication, IQ, and adaptive behaviors (Fisher and Meyer, 2002; Stahmer et al., 2011; Nahmias et al., 2014). Inclusion in mainstream education is also motivated by ethical considerations as a basic human right of children with ASD and their families (Allan, 2007).

In contrast, children with ASD who are placed in exclusive special education settings are likely to benefit from more intensive intervention programs that are managed by a larger number of more experienced staff and the availability of specialized facilities (Mesibov and Shea, 1996). Indeed, a large number of studies have demonstrated that young children with ASD who are placed in special education settings and receive intensive treatments exhibit significant improvements in cognitive and social abilities as 
well as adaptive skills (Harris et al., 1991; Rogers and Vismara, 2008; Zachor and Ben Itzchak, 2010; Talbott et al., 2016).

Despite the dramatic differences across educational settings, longitudinal studies have rarely compared their efficacy for ASD children with different characteristics. One recent study has reported that 3-5-year-old ASD children with lower adaptive and social-communication abilities made larger cognitive gains in inclusion programs as compared with ASD children placed in exclusive special education programs (Nahmias et al., 2014). However, large-scale longitudinal studies are lacking and the effectiveness of each educational setting remains unclear. This hinders the establishment of clinical guidelines and leaves parents to make somewhat arbitrary placement choices, particularly in situations where the child is young and both educational settings are equally accessible.

To date, several studies, mostly focusing on school-age children, have examined how the behavioral characteristics of ASD children influence educational placement decisions. While these studies have demonstrated that there is considerable overlap in the abilities and difficulties of children who are placed in both settings, older children who have more severe ASD symptoms, lower cognitive abilities, and more behavioral problems were more likely to be placed in special rather than mainstream education settings (Eaves and Ho, 1997a; White et al., 2007; Lauderdale-Littin et al., 2013; Towle et al., 2018; Rattaz et al., 2019). It is currently unknown whether similar differences are apparent in young 2-5-year-old children who are placed in special or mainstream education settings, immediately after receiving their ASD diagnosis.

In Israel, after receiving a formal diagnosis, parents of ASD children choose whether to place their child in a mainstream or special education setting. The Israeli Ministry of Education manages all mainstream and special education pre-school programs for children 3-years-old and over, which are freely available to all children. ASD children younger than 3-years-old are eligible for placement in a special education daycare center or allocation of an assistant that accompanies the child to a mainstream daycare center. All daycare centers in Israel are operated privately, but costs are subsidized by the Ministry of Welfare. Special education settings typically include only 8 ASD children and have a professional staff that includes special education pre-school teachers, 
speech therapists, occupational therapists, developmental psychologists, and other therapists. In contrast, mainstream education settings typically have 15-30 children and a limited staff of mainstream pre-school teachers who receive 1-2 hours of weekly guidance from a special education teacher.

In the current study we utilized a large population database managed by the National Autism Research Center of Israel to compare behavioral and socio-demographic characteristics of ASD children who were placed in either educational setting. We focused on their initial placement into daycare centers or pre-schools that took place immediately after receiving the ASD diagnosis.

\section{Methods:}

Participants: This retrospective study examined data from the National Autism Research Center (www.autismisrael.org). The center maintains a database with information from over 1000 ASD children who were diagnosed with ASD since 2015 in the southern district of Israel (Meiri et al., 2017). The majority of children return to the center for an annual follow-up, which includes the same behavioral tests carried out during initial diagnosis. From this population we selected a sample of 242 children who were 11-65-months-old (mean age $=33.7$ months), had completed an Autism Diagnostic Observation Schedule-2 (ADOS-2) assessment (Lord et al., 2012), and attended a follow-up meeting where parents completed a questionnaire with information regarding the child's initial educational placement. The sample included 100 children who were placed in daycare centers and 142 children who were placed in pre-school kindergartens (Tables 1\&2). The study was approved by the Soroka University Medical Center (SUMC) Helsinki committee and conforms to the declaration of Helsinki.

Procedures and Measures: All participating children completed an ADOS-2 assessment that was administered by a trained clinician with over 10 years of clinical experience. We used the total ADOS comparison scores as well as the relative Social Affect (SA) and Restricted and Repetitive Behavior (RRB) scores to compare the severity of ASD symptoms across children who completed different ADOS modules (Hus et al., 2014). Spoken language ability was estimated with the score of the A1 item on the ADOS 
Toddler module. This item lists five levels of spoken language ranging from 0 (language level appropriate to chronological age) to 4 (no use of significant words). In older children, we grossly estimated spoken language ability by comparing the number of children who were diagnosed with ADOS Module 1 (used with children who do not speak more than single words) versus Module 2 (used with children who speak in simple phrases). DSM-5 levels of support were indicated by an experienced physician (child neurologist or child psychiatrist) after a thorough clinical exam.

Cognitive scores were available for 171 of the children (70\% of the children). The remaining 71 children were unable to successfully complete cognitive testing due to lack of cooperation. Cognitive ability was measured using either the Bayley Scales of Infant and Toddler Development, Third Edition (Viezel et al., 2014) or the Wechsler Preschool and Primary Scale of Intelligence, Third Edition (Luiselli et al., 2013). Both tests yield equivalent standardized scores with a mean of 100 and a standard deviation of 15 .

Finally, parents of 204 participating children completed a background questionnaire that included socio-demographic questions about their education level and economic status.

Data analysis: All statistical analyses were performed using SPSS (IBM, USA), separately for the two age groups: daycare centers and pre-school kindergartens. We compared children in mainstream educational settings and children in special education settings using two-tailed t-tests (assuming un-equal variance), Mann-Whitney tests (for ordinal variables), and Chi-Square tests (for categorical variables). In all cases alpha was set to 0.05. In a final analysis we also performed a logistic regression using the ADOS-SA, ADOS-RRB, cognitive scores, and maternal education as predictors for placement choice in either setting.

\section{Results:}

We compared a variety of behavioral and socio-demographic characteristics between ASD children who were placed in special education and those in mainstream education (Tables 1\&2). Theses comparisons were performed separately for children in daycare centers (age 11-36 months) and those in pre-school kindergartens (age 32-60 months). 
Children in the daycare center groups were diagnosed at an earlier age (mean $=25.5$ months) than children in the pre-school groups (mean $=39.4$ months). Age of diagnosis, however, did not differ significantly between special and mainstream education settings. This was true both for the children in daycare centers ( $t(40.8)=0.8$, $p=0.43)$ and the children in pre-school kindergartens $(t(117.5)=1.36, p=0.17)$. This demonstrates that placement into special education or mainstream education was not influenced by the age of diagnosis (i.e. the time at which autism symptoms were first recognized).

\begin{tabular}{|c|c|c|c|c|c|}
\hline \multicolumn{6}{|c|}{ DAYCARE CENTERS } \\
\hline & $\begin{array}{l}\text { MAINSTREAM } \\
(\mathrm{N}=28, \mathrm{MALES}=17)\end{array}$ & $\begin{array}{l}\text { SPECIAL } \\
(\mathrm{N}=72, \text { MALES=52) }\end{array}$ & STATISTICS & $\mathbf{P}$ & COHEN'S D \\
\hline $\begin{array}{l}\text { AGE OF DIAGNOSIS } \\
\text { (MONTHS) }\end{array}$ & $26.3(s d=6.07)$ & $25.3(\mathrm{sd}=4.8)$ & $t(40.8)=0.8$ & 0.43 & 0.19 \\
\hline $\begin{array}{l}\text { ADOS COMPARISON } \\
\text { SCORES }\end{array}$ & $7.86(\mathrm{sd}=2.12)$ & $8.58(\mathrm{sd}=1.48)$ & $t(37.8)=-1.66$ & 0.1 & 0.39 \\
\hline $\begin{array}{l}\text { ADOS - SA } \\
\text { COMPARISON SCORES }\end{array}$ & $8.04(\mathrm{sd}=1.51)$ & $9.01(\mathrm{sd}=1.44)$ & $t(38.4)=-2.24$ & $0.03 *$ & 0.53 \\
\hline $\begin{array}{l}\text { ADOS - RRB } \\
\text { COMPARISON SCORES }\end{array}$ & $7.18(\mathrm{sd}=1.44)$ & $6.97(s d=1.38)$ & $t(47.5)=0.65$ & 0.52 & 0.15 \\
\hline DSM-SA & $\begin{array}{l}\text { Level 1: } n=0 \\
\text { Level2: } n=14(56 \%) \\
\text { Level3: } n=11(44 \%)\end{array}$ & $\begin{array}{l}\text { Level1: } n=2(2.9 \%) \\
\text { Level2: } n=21(30.9 \%) \\
\text { Level3 : } n=45(66.2 \%)\end{array}$ & $\chi 2(93)=5.29$ & 0.07 & \\
\hline DSM-RRB & $\begin{array}{l}\text { Level1: } n=3(12 \%) \\
\text { Level2: } n=14(56 \%) \\
\text { Level3: } n=8(32 \%)\end{array}$ & $\begin{array}{l}\text { Level1: } n=3(4.4 \%) \\
\text { Level2: } n=42(61.8 \%) \\
\text { Level3: } n=23(33.8 \%)\end{array}$ & $\chi 2(93)=1.75$ & 0.42 & \\
\hline COGNITIVE SCORES & $80.6(s d=9.65)$ & $74.1(s d=13.8)$ & $t(47.9)=2.14$ & $0.04 *$ & 0.54 \\
\hline MOTHER AGE AT BIRTH & $31(s d=6.13)$ & $30.7(\mathrm{sd}=5.87)$ & $t(48.5)=0.21$ & 0.83 & 0.05 \\
\hline FATHER AGE AT BIRTH & $35.1(\mathrm{sd}=8.8)$ & $34(s d=7.6)$ & $t(42.9)=0.52$ & 0.6 & 0.12 \\
\hline $\begin{array}{l}\text { MOTHER EDUCATION } \\
\text { (YEARS) }\end{array}$ & $14.5(\mathrm{sd}=3.03)$ & $12.8(\mathrm{sd}=2.46)$ & $t(42.1)=2.42$ & $0.02 *$ & 0.62 \\
\hline $\begin{array}{l}\text { FATHER EDUCATION } \\
\text { (YEARS) }\end{array}$ & $13.6(\mathrm{sd}=3.21)$ & $12(s d=1.27)$ & $t(27)=2.3$ & $0.03 *$ & 0.65 \\
\hline
\end{tabular}


Table 1: Behavioral and socio-demographic characteristics of ASD children who were placed in mainstream and special education daycare centers. Asterisks: significant differences across groups $p<0.05$, uncorrected.

\begin{tabular}{|c|c|c|c|c|c|}
\hline \multicolumn{6}{|c|}{ PRE-SCHOOL KINDERGARTENS } \\
\hline & $\begin{array}{l}\text { MAINSTREAM } \\
(\mathrm{N}=50, \mathrm{MALES}=38)\end{array}$ & $\begin{array}{l}\text { SPECIAL } \\
(\mathrm{N}=92, \mathrm{MALES}=74)\end{array}$ & STATISTICS & $\mathbf{P}$ & $\begin{array}{l}\text { COHEN'S } \\
\text { D }\end{array}$ \\
\hline $\begin{array}{l}\text { AVERAGE AGE OF } \\
\text { DIAGNOSIS (MONTHS) }\end{array}$ & $40.6(s d=7)$ & $38.8(\mathrm{sd}=8.5)$ & $\mathrm{t}(117.5)=1.36$ & 0.17 & 0.23 \\
\hline $\begin{array}{l}\text { ADOS COMPARISON } \\
\text { SCORES }\end{array}$ & $6.78(\mathrm{sd}=1.97)$ & $7.36(\mathrm{sd}=1.99)$ & $\begin{array}{l}t(101.6)=- \\
1.65\end{array}$ & 0.1 & 0.29 \\
\hline ADOS-SA & $6.62(\mathrm{sd}=1.97)$ & $7.25(\mathrm{sd}=2.24)$ & $t(112)=-1.72$ & 0.87 & 0.3 \\
\hline ADOS-RRB & $7.42(\mathrm{sd}=1.47)$ & $7.70(\mathrm{sd}=1.59)$ & $\begin{array}{l}t(107.8)=- \\
1.03\end{array}$ & 0.3 & 0.18 \\
\hline DSM-SA & $\begin{array}{l}\text { Level 1: } n=5(10.4 \%) \\
\text { Level2: } n=29(60.4 \%) \\
\text { Level3: } n=14(29.2 \%)\end{array}$ & $\begin{array}{l}\text { Level1: } n=7(7.9 \%) \\
\text { Level2: } n=37(41.6 \%) \\
\text { Level3: } n=45(50.6 \%)\end{array}$ & $\chi 2(137)=5.84$ & $0.05 *$ & \\
\hline DSM-RRB & $\begin{array}{l}\text { Level1: } n=7(14.6 \%) \\
\text { Level2: } n=36(75 \%) \\
\text { Level3: } n=5(10.4 \%)\end{array}$ & $\begin{array}{l}\text { Level1: } n=8(9 \%) \\
\text { Level2: } n=57(64 \%) \\
\text { Level3: } n=24(27 \%)\end{array}$ & $\chi 2(137)=5.47$ & 0.06 & \\
\hline COGNITIVE SCORES & $82.7(\mathrm{sd}=11.4)$ & $74.9(\mathrm{sd}=13.3)$ & $\mathrm{t}(99.3)=3.25$ & $0.002 *$ & 0.62 \\
\hline MOTHER AGE AT BIRTH & $32(s d=5.2)$ & $31(s d=5.7)$ & $\mathrm{t}(97.7)=0.91$ & 0.36 & 0.17 \\
\hline FATHER AGE AT BIRTH & $35.4(\mathrm{sd}=7)$ & $34.1(\mathrm{sd}=6.5)$ & $t(81.8)=0.97$ & 0.33 & 0.19 \\
\hline $\begin{array}{l}\text { MOTHER EDUCATION } \\
\text { (YEARS) }\end{array}$ & $14.5(\mathrm{sd}=3)$ & $12.8(\mathrm{sd}=2.5)$ & $t(78.8)=3.33$ & $0.001 *$ & 0.7 \\
\hline $\begin{array}{l}\text { FATHER EDUCATION } \\
\text { (YEARS) }\end{array}$ & $13.6(\mathrm{sd}=3.2)$ & $12(\mathrm{sd}=1.3)$ & $t(50.3)=1.7$ & 0.128 & 0.35 \\
\hline
\end{tabular}

Table 2: Behavioral and socio-demographic characteristics of ASD children who were placed in mainstream and special education pre-school kindergartens. Asterisks: significant differences across groups $p<0.05$, uncorrected.

\section{ADOS and DSM symptom severity}

We found minor differences in ADOS comparison scores and DSM severity levels across children in the special and mainstream groups (Tables 1\&2, Figure 1). There were no 
significant differences across groups in ADOS comparison scores of children in daycare centers $(\mathrm{t}(37.8)=-1.66, \mathrm{p}=0.1, \mathrm{~d}=0.39)$ or in pre-school kindergartens $(\mathrm{t}(101.6)=$ $1.65, p=0.1, d=0.3)$. When separating the ADOS scores into their SA and RRB components, we found significant differences across groups in the ADOS-SA scores of children in daycare centers $(\mathrm{t}(38.4)=-2.25, \mathrm{p}=0.03, \mathrm{~d}=0.53)$, but not in kindergartens $(t(107.8)=-1.72, p=0.087, d=0.3)$. There were no significant differences in ADOS-RRB scores of children in either group $(p>0.3)$. Note that these analyses were not corrected for multiple comparisons to increase sensitivity.

The levels of support that were assigned to each child by their physician according to DSM-5 criteria revealed minor, marginally significant, differences across groups.

Children in special education daycare centers exhibited a trend for requiring higher levels of social support $(\chi 2(93)=5.92, p=0.07)$, a difference that was significant across the kindergarten groups $(\chi 2(137)=5.84, p=0.05)$. Similar findings were also apparent in the support levels of the RRB symptoms of the kindergarten groups $(\chi 2(137)=5.47$, $p=0.07)$, but not the daycare groups $(\chi 2(93)=1.75, p=0.42)$. These analyses were also not corrected for multiple comparisons.

\section{Cognitive abilities}

Children in mainstream education exhibited significantly higher cognitive scores than those in special education (Tables 1\&2). This was true both for children in day care centers $(t(47.9)=2.14, p=0.04, d=0.54)$ and those in kindergartens $(t(99.3)=3.25, p$ $=0.002, d=0.63)$. Note that children in special education settings had, on average, cognitive scores that were 6.5 and 7.8 points lower than the children in mainstream education (in day care centers and kindergartens respectively). This corresponds to approximately half of one standard deviation in standardized cognitive scores (in all standardized cognitive assessments 1 standard deviation equals 15 points). Hence, while the differences were significant, their size was modest, with considerable overlap in cognitive scores across educational settings (Figure 1).

Almost $30 \%$ of the children (71 out of 242 ) were unable to complete the cognitive tests successfully, because they did not cooperate with the developmental psychologist who performed the test. The number of children who did not complete the cognitive tests did not differ significantly across day care groups $(\chi 2(100)=1.135, p=0.567)$, but was 
significantly larger in special education kindergartens in comparison to mainstream kindergartens $(\chi 2(142)=3.693, p=0.05)$. This suggests that cognitive differences across the kindergarten groups may be potentially larger than estimated, if one assumes that non-cooperative children tend to have low cognitive abilities.

A

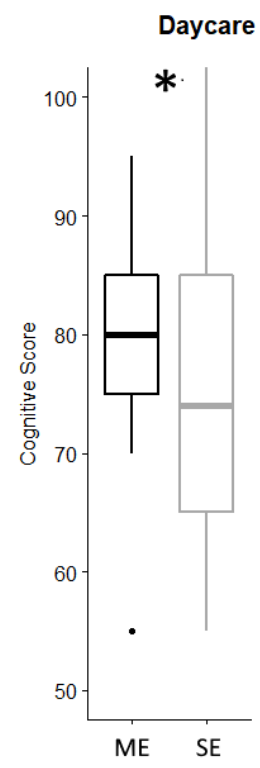

B

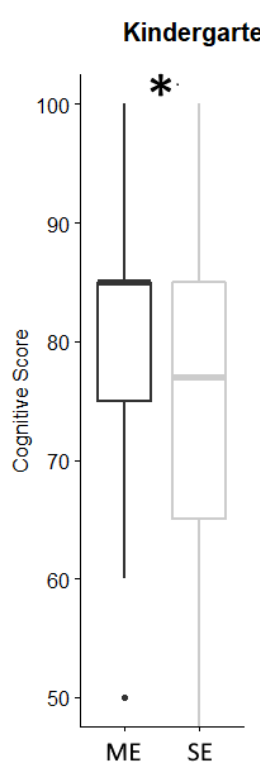

Figure 1: Cognitive and ADOS scores. A: Box plot figures demonstrate the distribution of cognitive and ADOS scores of ASD children who were placed in mainstream education (black) and special education (gray) day care centers. B: Cognitive and ADOS scores of ASD

children in pre-school kindergartens (same format as A). Asterisks: significant difference across groups ( $p<0.05$, two-tailed t-test). ME: mainstream education. SE: special education.

\section{Language}

We compared language abilities across groups using gross measures available from the ADOS assessments. The vast majority of ASD children who were in daycare centers were assessed with the ADOS toddler module. In this module, item A1 quantifies the spoken language abilities of the children (see Methods). When comparing the scores on this item, we did not find any significant differences in spoken language levels across children who were placed in mainstream and special education daycare centers (U (85) $=605, p=0.36)$.

All of the children in the pre-school kindergartens were assessed with modules 1 or 2 of the ADOS. The selection of module is indicative of spoken language abilities (i.e., module 2 requires higher language capabilities than module 1). There were significant differences in the module selected for assessments children placed in special versus 
mainstream kindergartens $(\chi 2(122)=10.63, p=0.001)$. Specifically, more children with higher language abilities were placed in mainstream kindergartens (Figure 2).

A

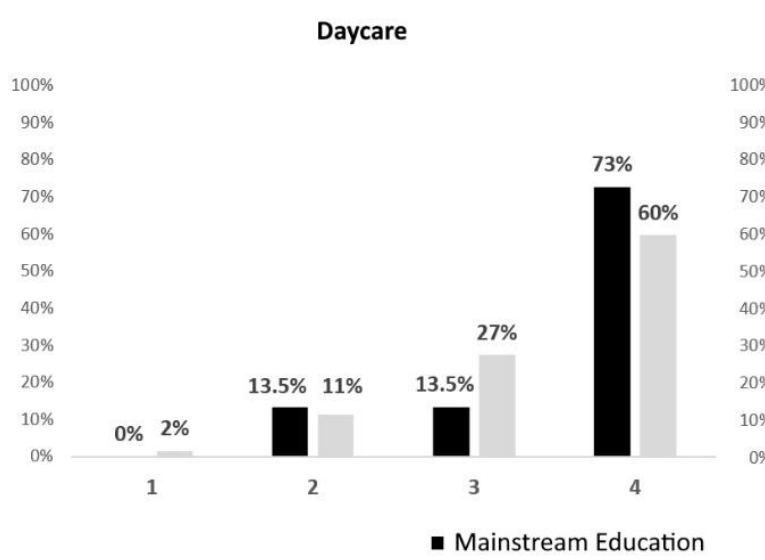

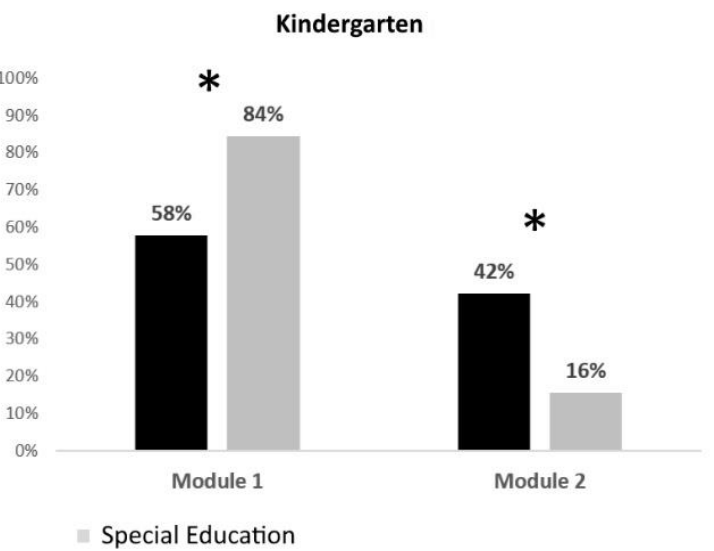

Figure 2: Spoken language levels. A: Language scores from item A1 of the ADOS toddler module, which was used to assess most of the children placed in daycare centers. B: Percentage of children in pre-school kindergartens who were assessed using module 1 (lower language abilities) versus module 2 (higher language abilities) of the ADOS. Black: mainstream education. Gray: special education (gray). Asterisks: significant difference across groups ( $p<0.05$, Chi-Square test).

\section{Parents' socio-demographic characteristics}

Parents' age at birth did not differ across groups of children in day care centers (mothers: $\mathrm{t}(48.5)=0.21, \mathrm{p}=0.83$; fathers: $\mathrm{t}(42.9)=0.52, \mathrm{p}=0.6$ ) or kindergartens (mothers: $\mathrm{t}(97.7)=0.91, \mathrm{p}=0.36$; fathers: $\mathrm{t}(81.8)=0.97, \mathrm{p}=0.33$ ).

Parents of children in mainstream education, however, had more years of education. This was apparent for mothers and fathers of children in day care centers (mothers: $t$ $(42.1)=2.52, p=0.02, d=0.62$; fathers: $t(27)=2.3, p=0.03, d=0.65)$ and for mothers of children in kindergartens (mothers: $t(78.8)=3.33, p=0.001, d=0.7$; fathers: $t(50.3)=$ $1.7, p=0.128, d=0.35)$.

\section{Characteristics that predict educational placement}


We tested whether we could predict initial educational placement in special or mainstream settings using several logistic regression analyses. First, we attempted to predict placement based on the cognitive and ADOS scores of all children from both age groups (daycare centers and kindergartens) who had these data $(n=173)$. The resulting regression model was significant $(\chi 2(3)=19.63, p<0.001)$, with a pseudo $R-$ square (Cox $\&$ Snell method) of 0.11 , and two significant predictors: ADOS-SA $(\beta=0.18$, $p=0.016)$ and cognitive $(\beta=-0.04, p=0.003)$. In a second model, we also added maternal years of education as an additional predictor $(n=118)$. The resulting model was also significant $(\chi 2(3)=17.28, p=0.002)$, with a pseudo R-square (Cox \& Snell method) of 0.14 , and two significant predictors: ADOS-SA $(\beta=0.19, p=0.046)$ and maternal education $(\beta=-0.22, p=0.01)$.

While the regression models described above were able to explain a significant amount of variability in placement choices (i.e., the models performed significantly better than chance), over $85 \%$ of the variance in placement choices remained unaccounted for. This suggests that the vast majority of placement choices are performed in a mostly arbitrary manner that has little to do with the child's abilities or needs.

\section{Discussion}

The results of this study suggest that initial placement of young children with ASD in mainstream or special education settings is performed in a somewhat arbitrary manner. While children in special education settings had significantly lower cognitive scores, the effect size of this finding was modest (Tables 1\&2) and there was considerable overlap in the cognitive abilities of ASD children across educational settings (Figure 1). Differences in autism severity across educational settings were also remarkably weak, with no significant differences in total ADOS scores across educational settings. However, children in special education daycare centers exhibited significantly lower ADOS-SA scores (Table 1) and children in special education kindergartens exhibited significantly higher DSM-SA support requirements (Table 2). Finally, language abilities did not differ significantly in the daycare groups, but were significantly lower in ASD children placed in special education kindergartens.

While the age of the parents did not differ significantly across educational settings, parental education (i.e., years of education) was significantly higher in children placed 
in mainstream education. Indeed, the difference in maternal education across educational settings had the largest effect size of any of the examined variables. Nevertheless, even when incorporating maternal education, cognitive scores, and ADOS scores into a single logistic regression model, the variance explained (pseudo Rsquared) remained remarkably low $(<15 \%)$. This demonstrated that the examined behavioral and demographic characteristics were poor predictors of early placement decisions.

\section{Educational placement and cognitive abilities}

Several studies have reported that lower cognitive abilities are associated with higher likelihood of placement in special as opposed to mainstream educational settings. This was reported for school-aged children in the United States (White et al., 2007), Canada (Eaves and Ho, 1997b), Singapore (Aljunied and Frederickson, 2011), and France (Rattaz et al., 2019). Indeed, first grade teachers consider cognitive abilities as the most important behavioural characteristic when making recommendations about placement decisions (Segall and Campbell, 2014).

Our results extend these findings, to some degree, to earlier ages and the initial placement decision made immediately after receiving the ASD diagnosis. Cognitive scores were indeed significantly lower in ASD children who were placed in special versus mainstream education settings (Tables 1\&2). However, the difference across settings, on average, was only 6.5 and 7.8 points in daycare centers and kindergartens, respectively. This corresponds to approximately half of one standard deviation in standardized cognitive scores. In contrast, previous studies with older children have reported much larger differences of, on average, 12 (White et al., 2007), 25 (Aljunied and Frederickson, 2011), and 30 (Eaves and Ho, 1997b) points. Cognitive differences across special and mainstream education settings are, therefore much weaker at early ages with considerable overlap in cognitive scores across groups, particularly in the youngest children who are attending day care (Figure 1).

\section{Educational placement and language abilities}

To the best of our knowledge, previous studies have not examined the impact of language abilities on educational placement. To address this point, we examined difference in gross language abilities as identified by the clinician who administered the 
ADOS assessments. This data revealed that language abilities may indeed have an impact on the placement choice of the older children in the kindergarten groups such that children with better language abilities were more often placed in mainstream education (Figure 2B). However, language abilities did not seem to have an impact on placement choices for the younger children who were similarly distributed in special and mainstream day care centers (Figure 2A). A more systematic and sensitive assessment of language differences across the two educational settings, using standardized language tests that yield expressive and receptive language scores, is highly warranted.

\section{Educational placement and autism severity}

Our results are in line with findings from previous studies, which have reported no statistically significant differences in total ADOS scores across educational settings (White et al., 2007). When specifically examining social behaviors, one previous study have reported significant differences in Vineland communication and socialization scores (Rattaz et al., 2019), but this was not found in another study (White et al., 2007). Our findings demonstrate that ADOS scores did not differ significantly across special and mainstream education groups, except for the ADOS-SA scores of the day care children (Tables 1\&2). There was indeed considerable overlap in ADOS scores across the educational settings, suggesting that autism severity has relatively little impact on early placement decisions (Figure 1).

\section{Educational placement and parental education}

In Israel, children who are placed in mainstream education do not receive treatments from speech and occupational therapists in their educational setting. Thus, parents have to arrange and coordinate these services on their own, often incurring additional costs. In contrast, children placed in special education settings receive such services within the educational setting. Our findings show that parent education was significantly higher for ASD children placed in mainstream education. Similar findings were reported in previous studies in France (Rattaz et al., 2019) and the U.S. (Kurth et al., 2016.) We speculate that more educated parents, who are likely to have larger financial abilities, are more likely to choose mainstream education setting given their socio-economical ability to organize and fund essential treatments outside the 
educational setting. Additional studies examining this issue are highly warranted for equating the opportunities given to parents with different socio-economical abilities.

\section{Limitations}

A major limitation of the current study is that we did not measure several behavioral characteristics that are likely to have an impact on placement decisions of ASD children. These include adaptive behaviors, which tend to be lower, and aberrant behaviors, which tend to be higher, in ASD children placed in special education settings (White et al., 2007; Rattaz et al., 2019). Integrating these and other measures of sensory sensitivities, medical comorbidities, anxiety, and language capabilities may reveal important differences across children in the two educational settings.

\section{Conclusions}

The uniqueness of this study was its focus on the initial educational placement of a relatively large group $(n=242)$ of young children with ASD. Furthermore, this is the first study to systematically examine placement choices in Israel. The results reveal some similarities with previous studies of older, school-aged, ASD children, including significantly higher cognitive scores in children who are placed in mainstream education setting. Differences across the educational settings, however, were dwarfed by the remarkable overlap in behavioral abilities of ASD children placed in either setting. These findings highlight the lack of clinical guidelines regarding initial educational placement and demonstrate that placement decisions are made in a somewhat arbitrary manner. Longitudinal studies using additional standardized measures of adaptive behaviors, language abilities, sensory sensitivities, and challenging behaviors are highly warranted for determining the efficacy of initial educational settings for children with different abilities and difficulties.

\section{References}

Aljunied M, Frederickson N (2011) Cognitive indicators of different levels of special educational support needs in autism. Res Autism Spectr Disord 5:368-376.

Allan J (2007) Rethinking Inclusive Education: The Philosophers of Difference in Practice 
Ashenfelter, O. \& Rouse, C. (2000). Schooling, intelligence and income in America. In: Arrow, K., Bowles, S., \& Durlauf, S. (Eds.). Meritocracy and economic inequality, Princeton: New Jersey: Princeton University Press.

Chasson GS, Harris GE, Neely WJ (2007) Cost comparison of early intensive behavioral intervention and special education for children with autism. J Child Fam Stud 16:401-413.

Eaves LC, Ho HH (1997a) School Placement and Academic Achievement in Children with Autistic Spectrum Disorders. J Dev Phys Disabil.

Eaves LC, Ho HH (1997b) School Placement and Academic Achievement in Children with Autistic Spectrum Disorders. J Dev Phys Disabil 9:277-291.

Fisher M, Meyer LH (2002) Development and social competence after two years for students enrolled in inclusive and self-contained educational programs. Res Pract Pers with Sev Disabil. Harris SL, Handleman JS, Gordon R, Kristoff B, Fuentes F (1991) Changes in Cognitive and Language Functioning of Preschool Children with Autism.

Harrower JK, Dunlap G (2001) Including children with autism in general education classrooms: A review of effective strategies. Behav Modif 25:762-784.

Hus V, Gotham K, Lord C (2014) Standardizing ADOS domain scores: Separating severity of social affect and restricted and repetitive behaviors. J Autism Dev Disord.

Kurth JA, Mastergeorge AM, Paschall K (n.d.) Economic and Demographic Factors Impacting Placement of Students with Autism.

Lauderdale-Littin S, Howell E, Blacher J (2013) Educational Placement for Children with Autism Spectrum Disorders in Public and Non-Public School Settings: The Impact of Social Skills and Behavior. Educ Train Autism Dev Disabil.

Lord C, Rutter M, Di Lavore P, Risi S, Gotham K, Bishop S (2012) Autism and Diagnostic Observation Schedule, Second Edition (ADOS-2) Manual (Part I): Modules 1-4.

Luiselli J et al. (2013) Wechsler Preschool and Primary Scale of Intelligence. In: Encyclopedia of Autism Spectrum Disorders.

Meiri G, Dinstein I, Michaelowski A, Flusser H, llan M, Faroy M, Bar-Sinai A, Manelis L, Stolowicz D, Yosef LL, Davidovitch N, Golan H, Arbelle S, Menashe I (2017) Brief Report: The Negev Hospital-University-Based (HUB) Autism Database. J Autism Dev Disord.

Mesibov GB, Shea V (1996) Full inclusion and students with autism. J Autism Dev Disord. 
Nahmias AS, Kase C, Mandell DS (2014) Comparing cognitive outcomes among children with autism spectrum disorders receiving community-based early intervention in one of three placements. Autism 18:311-320.

Rattaz C et al. (2019) School Inclusion in Children and Adolescents with Autism Spectrum Disorders in France: Report from the ELENA French Cohort Study. J Autism Dev Disord.

Ravet J (2011) Inclusive/exclusive? perspectives on autism and inclusion: the case for an integrative position. Int J Incl Educ 15:667-682

Rogers SJ, Vismara LA (2008) Evidence-based comprehensive treatments for early autism. J Clin Child Adolesc Psychol 37:8-38 Available at: http://www.ncbi.nlm.nih.gov/pubmed/18444052 [Accessed February 17, 2020].

Segall MJ, Campbell JM (2014) Factors influencing the educational placement of students with autism spectrum disorders. Res Autism Spectr Disord 8:31-43.

Stahmer AC, Akshoomoff N, Cunningham AB (2011) Inclusion for toddlers with autism spectrum disorders: The first ten years of a community program. Autism 15:625-641

Talbott MR, Estes A, Zierhut C, Dawson G, Rogers SJ (2016) Early Start Denver Model. In, pp 113-149.

Towle PO, Vacanti-Shova K, Higgins-D'Alessandro A, Ausikaitis A, Reynolds C (2018) A Longitudinal Study of Children Diagnosed with Autism Spectrum Disorder Before Age Three: School Services at Three Points Time for Three Levels of Outcome Disability. J Autism Dev Disord 48:3747-3760

Viezel K, Zibulsky J, Dumont R, Willis JO (2014) Bayley Scales of Infant and Toddler Development, Third Edition. In: Encyclopedia of Special Education.

White SW, Scahill L, Klin A, Koenig K, Volkmar FR (2007) Educational placements and service use patterns of individuals with autism spectrum disorders. J Autism Dev Disord.

Yianni-Coudurier C, Darrou C, Lenoir P, Verrecchia B, Assouline B, Ledesert B, Michelon C, Pry R, Aussilloux C, Baghdadli A (2008) What clinical characteristics of children with autism influence their inclusion in regular classrooms? J Intellect Disabil Res 52:855-863.

Zachor DA, Ben Itzchak E (2010) Treatment approach, autism severity and intervention outcomes in young children. Res Autism Spectr Disord 4:425-432. 